\title{
A pharmacodynamic analysis of resistance trends in pathogens from patients with infection in intensive care units in the United States between 1993 and 2004
}

\author{
Kathryn J Eagye1, David P Nicolau*1,2, Shawn R Lockhart³, John P Quinn ${ }^{4,5,6}$, \\ Gary V Doern ${ }^{3}$, Gale Gallagher ${ }^{7}$ and Murray A Abramson ${ }^{7}$
}

Address: ${ }^{1}$ Center for Anti-Infective Research and Development, Hartford Hospital, 80 Seymour Street, Hartford, CT 06102, USA, ${ }^{2}$ Division of Infectious Diseases, Hartford Hospital, 80 Seymour Street, Hartford, CT 06102 USA, ${ }^{3}$ Div. of Clinical Microbiology, University of Iowa Hospital and Clinics, 200 Hawkins Drive, Iowa City, IA 52242, USA, ${ }^{4}$ John H. Stroger Hospital, 1900 West Polk Street, Chicago, IL 60612, USA, ${ }^{5}$ Chicago Infectious Disease Research Institute, 1650 Harrison Street, Chicago, IL 60612, USA, ${ }^{\circ}$ Rush University Medical Center, 1650 Harrison Street, Chicago, IL 60612, USA and ${ }^{7}$ Merck Research Laboratories, Merck \& Co., 126 E Lincoln Ave., Rahway, NJ 07065, USA

Email: Kathryn J Eagye - keagye@harthosp.org; David P Nicolau* -dnicola@harthosp.org; Shawn R Lockhart - shawn-lockhart@uiowa.edu; John P Quinn - esblman@yahoo.com; Gary V Doern - gary-doern@uiowa.edu; Gale Gallagher - gale_gallagher@merck.com;

Murray A Abramson - murray_abramson@merck.com

* Corresponding author

Published: I October 2007

Annals of Clinical Microbiology and Antimicrobials 2007, 6:1I doi:10.1 I86/1476-07II-6II

This article is available from: http://www.ann-clinmicrob.com/content/6/I/II

(c) 2007 Eagye et al; licensee BioMed Central Ltd.

This is an Open Access article distributed under the terms of the Creative Commons Attribution License (http://creativecommons.org/licenses/by/2.0), which permits unrestricted use, distribution, and reproduction in any medium, provided the original work is properly cited.
Received: 17 July 2007

Accepted: I October 2007

\begin{abstract}
Background: Increasing nosocomial pathogen resistance to available antimicrobial agents is of growing concern. While higher MICs can diminish antimicrobial effectiveness, dose adjustments often mitigate this effect. This study's objective was to ascertain whether MICs among major pathogens in the ICU to several commonly used agents have increased enough to significantly impact their ability to achieve bactericidal effect.
\end{abstract}

Methods: Cefepime, ceftriaxone, imipenem and piperacillin-tazobactam MICs were determined with 74,394 Gram-negative bacilli obtained from ICU patients with various infections in the US between 1993 and 2004. Results were grouped into four 3-year periods. The predicted cumulative fraction of response (CFR) was estimated based on patient-derived pharmacokinetic values and Monte Carlo simulation. Trends in CFR over the four study periods were assessed using the Cochran-Armitage test. The primary analysis included all organisms combined; Pseudomonas aeruginosa and Acinetobacter species were also evaluated individually.

Results: In the primary analysis, imipenem $500 \mathrm{mg}$ q6h showed CFRs from $87 \%$ to $90 \%$ across all four study periods, with a trend toward slightly improved bactericidal target attainment $(p<0.01)$. CFRs for cefepime $2 \mathrm{~g} q \mathrm{I} 2 \mathrm{~h}$ and piperacillin-tazobactam $4.5 \mathrm{~g}$ q6h both declined by $2 \%(\mathrm{p}<0.0 \mathrm{I}$ and $p<0.05$, respectively), reflecting upward shifts in the underlying MIC distributions. Ceftriaxone had $<52 \%$ CFR for all regimens in all periods, with no significant trend. Against $P$. aeruginosa, significant declines in CFR were seen for (range, $p$-value): imipenem I g q8h (82\%-79\%, $p<0.01$ ), cefepime I g q $2 \mathrm{~h}(70 \%-67 \%, \mathrm{p}<0.0 \mathrm{l})$, cefepime $2 \mathrm{~g}$ q $2 \mathrm{~h}(84 \%-82 \%$, $\mathrm{p}<0.05)$, piperacillintazobactam 3.375 g q6h (76\%-73\%, p < 0.0 I), piperacillin-tazobactam 4.5 g q8h $(71 \%-68 \%$, p < $0.0 \mathrm{I})$, and piperacillin-tazobactam $4.5 \mathrm{~g} \mathrm{q} 6 \mathrm{~h}(80 \%-77 \%, \mathrm{p}<.0 \mathrm{l})$. Against Acinetobacter spp., all 
regimens of imipenem, cefepime and piperacillin-tazobactam showed significant declines in CFR over time $(p<0.01)$.

Conclusion: Our observations suggest that as a result of increasing antimicrobial resistance among ICU pathogens in the US, drug effectiveness, assessed as a function of individual agents' ability to attain pharmacodynamic targets, has declined, especially with $P$. aeruginosa and Acinetobacter spp. Cefepime $2 \mathrm{~g} \mathrm{q8h}$ and imipenem were the most potent agents against these species, respectively. More aggressive dosing of all of the agents characterized could preserve their clinical utility, but this must be balanced with safety and tolerability issues by the physician.

\section{Introduction}

Surveillance studies have revealed increasing rates of resistance among bacteria commonly implicated in serious hospital infections; resistant pathogens are associated with higher mortality rates than are susceptible organisms[1,2]. As the pipeline of new antimicrobial agents for Gram-negative pathogens shrinks, the longevity of existing compounds becomes a matter of primary concern[3].

Our earlier work has shown that pharmacokinetic/pharmacodynamic (PK/PD) modeling based on Monte Carlo simulations can be used reliably to predict the ability of antimicrobial regimens to achieve maximum bactericidal effect against organisms implicated in nosocomial infections [4]. Further, we have demonstrated that alterations in dose can extend the coverage of many current compounds $[5,6]$.

Using Monte Carlo analyses, the objective of the current study was to use PK/PD modeling to assess the profile of activity of four antimicrobial agents commonly used to treat serious infections - imipenem, ceftriaxone, cefepime and piperacillin-tazobactam - versus a large collection of bacteria recovered from patients in the intensive care unit (ICU) setting in the United States between 1993 and 2004. These organisms had been characterized as part of the Merck Intensive Care Unit Surveillance Survey (ISS) Program. Secondly, we attempted to determine if the activity profile of any of these antimicrobial agents had diminished over time. A third objective was to determine the effect of dose selection on the activity profile of the agents.

\section{Methods}

This investigation employed Monte Carlo simulation techniques to estimate the relative probability that various antimicrobial agents would achieve maximally effective (i.e. bactericidal) exposures against isolates of Gram-negative bacilli recovered from patients with infection in the ICU. A PK model was developed for each compound and then used in a simulation to incorporate patient variability. The model, the input parameters, and the simulation technique are described below. The following antibiotic regimens were examined (administered as 30-minute intravenous infusions): cefepime 1 gram ( $1 \mathrm{~g}$ ) every twelve hours (q12h), $2 \mathrm{~g} \mathrm{q} 12 \mathrm{~h}$ and $2 \mathrm{~g} \mathrm{q} 8 \mathrm{~h}$; ceftriaxone 1 $\mathrm{g} \mathrm{q} 24 \mathrm{~h}$ and $2 \mathrm{~g} \mathrm{q} 24 \mathrm{~h}$; imipenem $500 \mathrm{mg} \mathrm{q} 6 \mathrm{~h}$ and $1 \mathrm{~g} \mathrm{q} 8 \mathrm{~h}$; and piperacillin/tazobactam $3.375 \mathrm{~g}$ q6h, $4.5 \mathrm{~g} \mathrm{q} 8 \mathrm{~h}$ and $4.5 \mathrm{~g} \mathrm{q6h}$.

\section{Microbiology}

MICs for bacterial isolates used in the analysis were obtained from the ISS Program from 1993 to 2004. A total of 44 different species were characterized. The 11 most commonly recovered species (which account for $94 \%$ of the tested population) are displayed in Table 1. For each compound, MIC results from all organisms tested were grouped to form one MIC frequency distribution for four intervals of three years each: 1993-1995, 1996-1998, 1999-2001, and 2002-2004. The MIC distributions were incorporated into the analysis as described in the following section, "Cumulative Fraction of Response." This scenario (all isolates included) was modeled in the primary analysis. Additionally, MIC distributions for two individual species, $P$. aeruginosa and Acinetobacter spp, were similarly divided into four intervals of the same 3-year periods. These groupings were used for sub-analyses of those species.

Table I: Eleven (I I) most commonly recovered species (constituting $\mathbf{9 4 \%}$ of the tested population) by frequency extracted from the Merck ICU Surveillance Program from 1993 to 2004 (Total of all isolates: 74,394).

\begin{tabular}{lcc}
\hline Organism & Number (\%) & Cumulative frequency \\
\hline Pseudomonas aeruginosa & $16,482(22)$ & $22.2 \%$ \\
Escherichia coli & $13,961(19)$ & $40.9 \%$ \\
Klebsiella pneumoniae & $10,571(14)$ & $55.1 \%$ \\
Enterobacter cloacae & $6,779(9)$ & $64.2 \%$ \\
Acinetobacter spp. & $4,642(6)$ & $70.5 \%$ \\
Serratia marcescens & $4,112(6)$ & $76.0 \%$ \\
Enterobacter aerogenes & $3,307(4)$ & $80.5 \%$ \\
Stenotrophomonas maltophilia & $3217(4)$ & $84.8 \%$ \\
Proteus mirabilis & $3,011(4)$ & $88.8 \%$ \\
Klebsiella oxytoca & $2,018(3)$ & $91.5 \%$ \\
Citrobacter freundii & $1,483(2)$ & $93.5 \%$ \\
\hline
\end{tabular}




\section{Pharmacokinetic model}

A two-compartment multiple dose model was developed to determine the 24-hour concentration-time profile at steady-state for each drug regimen. PK parameters were used as input variables, and included total body clearance $\left(C L_{T}\right)$, volume of the central compartment $\left(\mathrm{V}_{c}\right)$, and the microtransfer rate constants between the central and peripheral compartments $\left(k_{12}\right.$ and $\left.k_{21}\right)$. Estimates of unbound fraction for each drug $\left(f_{u}\right)$ were also used as inputs.

PK parameters for each compound under study were obtained from previously published population PK studies in critically ill patients, except for $f_{u^{\prime}}$ which was derived from the package insert for each drug; the mean and standard deviation for each of these parameters have been previously published[5,7]. Covariance matrices were either reported in or were calculated from these studies and were applied to the input pharmacokinetic variables used in the simulations.

\section{Monte Carlo simulation}

A 5,000 trial Monte Carlo simulation (Crystal Ball 2000, Decisioneering, Denver, CO, USA) was conducted for each antimicrobial regimen using the pharmacokinetic model in order to determine the regimen's probability of target attainment (PTA) profile. PTA is the probability that the regimen will meet or exceed a pre-defined pharmacodynamic target at a given MIC dilution[8]. Here, PTA is the proportion of the 5,000 trials in each simulation that achieved the target level of a PD index at each MIC in doubling dilutions from 0.008 to $128 \mu \mathrm{g} / \mathrm{ml}$.

The target indices were selected based on the PD properties (i.e., time-dependent or concentration-dependent killing) of the compound. As the four compounds modeled here exhibit time-dependent killing, the proportion of the dosing interval during which the concentration of free (unbound) drug remains above the MIC is the appropriate PD index[9]. For the carbapenems (including imipenem), 40\% f $>$ MIC was considered bactericidal; $50 \%$ $f \mathrm{~T}>\mathrm{MIC}$ was considered bactericidal for piperacillin-tazobactam and for the cephalosporins $[9,10]$. The PTA profile was then determined for each regimen.

\section{Cumulative Fraction of Response}

CFR is the probability that the regimen will attain its PD index against the specific population of organisms characterized in the ISS Program. Each regimen's simulationderived PTA is multiplied by the percentage of isolates found at each MIC dilution; the sum of these products is the CFR[8]. For each scenario (all isolates in the primary analysis or an individual species in the sub-analyses), this process was applied to four MIC distributions, one from each three-year interval, to obtain the CFR for each regimen during each interval.

A CFR of $90 \%$ was considered to be the threshold for achieving reliable empiric therapy[11]. Confidence intervals were calculated around each CFR result to provide a measure of statistical significance; however, it is important to consider these results in light of clinical significance as well. The CFR for each interval can be compared to illustrate trends in the ability of each regimen to achieve its best killing effect. A p-value $\leq 0.05$ indicates a statistically significant trend across the four periods, where lower CFRs in later periods reflect a decline in activity (higher CFRs would indicate an improvement). Confidence intervals around each CFR probability were calculated at $\alpha=0.05$ using the Newcombe-Wilson method without correction for continuity[12]. Trends in cumulative fraction of response for each regimen were assessed using the Cochran-Armitage test for trend (SAS version 9.0, Cary, NC, USA).

\section{Results}

Results of the primary analysis are presented in Table 2. Against all species aggregated, imipenem demonstrated a trend toward slightly improving activity over the four periods, and had predicted responses near 90\%. Cefepime displayed a decreasing activity profile over time, though still showing generally high predicted responses (80\%-90\%, depending on dosing regimen). Ceftriaxone had relatively low predicted response rates $(<52 \%)$ across all periods, and no significant trend was detected. Piperacillin-tazobactam $4.5 \mathrm{~g}$ q6h displayed the highest predicted response among piperacillin-tazobactam regimens (80$78 \%$ ), with a trend toward decreasing activity. The less aggressive piperacillin-tazobactam regimens $(4.5 \mathrm{~g} \mathrm{q} 8 \mathrm{~h}$ and $3.375 \mathrm{~g} \mathrm{q6h)}$ displayed lower CFRs and were stable to trend.

Sub-analyses were conducted on two species which have been shown in the literature to be highly pathogenic and whose resistance is of current concern: the results for $P$. aeruginosa and Acinetobacter spp. are displayed in Tables 3 and 4, respectively. Against both species, all active agents showed significant declines in predicted CFR over time. For $P$. aeruginosa, the greatest activity was observed with higher doses of cefepime (particularly $2 \mathrm{~g}$ q8h). Against Acinetobacter spp., imipenem displayed the most favorable activity profile, with predicted CFRs $>20 \%$ higher than cefepime and piperacillin-tazobactam.

\section{Discussion}

We examined nosocomial isolates collected from ICUs in the United States during a multi-year surveillance study in order to assess the performance of antibiotics commonly used to treat infections in this setting, and to identify any 
Table 2: Cumulative fraction of response for various drug regimens against MICs collected from the Merck ICU Surveillance Study (ISS) Program, in four time intervals.

\begin{tabular}{|c|c|c|c|c|c|}
\hline \multirow{2}{*}{$\begin{array}{l}\text { Antimicrobial agent: } \\
\text { Time interval: }\end{array}$} & \multicolumn{5}{|c|}{ CFRa (\%) } \\
\hline & $1993-1995 b$ & $1996-1998$ & $|999-200|$ & 2002-2004 & $\mathrm{p}$-value \\
\hline Imipenem 500 mg q6h & 87.0 & 87.7 & 88.2 & 90.6 & $<.0001$ \\
\hline Imipenem I g q8h & 86.8 & 87.3 & 87.9 & 90.2 & $<.0001$ \\
\hline Ceftriaxone I g q24h & 32.5 & 30.9 & 32.3 & 31.5 & .6128 \\
\hline Ceftriaxone 2 g q24h & 51.6 & 49.2 & 51.2 & 50.0 & .3726 \\
\hline Cefepime I g q $2 \mathrm{~h}$ & - & 80.6 & 80.4 & 78.6 & .0118 \\
\hline Cefepime $2 \mathrm{~g}$ q $2 \mathrm{~h}$ & - & 86.8 & 87.5 & 84.8 & .0027 \\
\hline Cefepime 2 g q8h & - & 91.8 & 93.5 & 89.9 & .0006 \\
\hline Pip-Tazo 3.375 g q6h & - & 76.6 & 77.3 & 75.1 & .0667 \\
\hline Pip-Tazo 4.5 g q8h & - & 72.1 & 72.9 & 70.9 & .1569 \\
\hline Pip-Tazo 4.5 g q6h & - & 79.4 & 79.9 & 77.5 & .0175 \\
\hline
\end{tabular}

aCFRs were calculated at 40\% $\mathrm{T}$ T>MIC for imipenem and 50\% $\mathrm{fT}>\mathrm{MIC}$ for the other compounds.

bNo data for cefepime or piperacillin-tazobactam (pip-tazo) was provided for 1993-1995.

trends in antimicrobial performance over time. Four broad-spectrum agents were studied: cefepime, ceftriaxone, imipenem and piperacillin-tazobactam. We used PK/ PD principles to predict the likelihood that each compound and dose would achieve its maximum bactericidal effect against the tested population of organisms, as measured by cumulative fraction of response. The Monte Carlo simulation technique enables us to predict the microbiological performance of different regimens while accounting for the variance in $\mathrm{PK}$ characteristics that such regimens are likely to encounter in critically ill patients. It should be noted that microbiological success is but one part of clinical success in treating infection, as many factors - co-morbidities, immunocompetence, etc. - contribute to the ultimate recovery of a patient. However, we have found that simulation-predicted microbiological response does indeed correlate with clinical response[13]. As such, identifying trends in probable microbiological success can provide useful insight into the clinical implications of changing resistance. We found moderate to good predicted responses ( $80 \%$ CFR or better) to all species aggregated as a group for three of the four compounds examined, with low response rates predicted for ceftriaxone. Response rates were dose-dependent, with more aggressive doses yielding greater predicted response. As to trend, imipenem's ability to achieve bactericidal effect showed a slight statistical improvement over time, whereas the effect of shifting MIC distributions on the other compounds was reflected in stable or worsening activity.

The examination of antibiotic performance against the entire cohort of isolates in the ISS Program is useful as a broad evaluation of continued efficacy to pathogens encountered in the ICU; however, clinically significant trends in any one species may be masked by the trend (or

Table 3: Cumulative fraction of response for various drug regimens against $P$. aeruginosa MICs collected from the Merck ICU Surveillance Study (ISS) Program, in four time intervals.

\begin{tabular}{|c|c|c|c|c|c|}
\hline \multirow{2}{*}{$\begin{array}{l}\text { Antimicrobial agent: } \\
\text { Time interval: }\end{array}$} & \multicolumn{5}{|c|}{$\mathrm{CFR}^{\mathrm{a}}(\%)$} \\
\hline & $1993-1995^{b}$ & $1996-1998$ & $|999-200|$ & 2002-2004 & $\mathrm{P}$-value \\
\hline Imipenem 500 mg q6h & 81.6 & 81.9 & 79.5 & 78.5 & $<.0001$ \\
\hline Imipenem I g q8h & 81.9 & 82.0 & 79.8 & 78.7 & $<.0001$ \\
\hline Ceftriaxone I g q24h & $\mathrm{I} .4$ & $\mathrm{I} .4$ & $\mathrm{I} .4$ & 1.3 & .6217 \\
\hline Ceftriaxone $2 \mathrm{~g}$ q24h & 3.2 & 3.2 & 3.1 & 2.8 & .2903 \\
\hline Cefepime I g q I $2 \mathrm{~h}$ & - & 69.9 & 68.3 & 67.2 & .0035 \\
\hline Cefepime $2 \mathrm{~g} q 12 \mathrm{~h}$ & - & 83.5 & 82.9 & 81.8 & .0240 \\
\hline Cefepime $2 \mathrm{~g}$ q8h & - & 92.2 & 92.7 & 91.2 & .0842 \\
\hline Pip-Tazo 3.375 g q6h & - & 75.9 & 74.0 & 73.3 & .0024 \\
\hline Pip-Tazo 4.5 g q8h & - & 70.8 & 69.0 & 68.4 & .0095 \\
\hline Pip-Tazo 4.5 g q6h & - & 79.6 & 77.5 & 76.7 & .0004 \\
\hline
\end{tabular}

aCFRs were calculated at $40 \% f \mathrm{~T}>\mathrm{MIC}$ for imipenem and $50 \% \mathrm{fT}>\mathrm{MIC}$ for the other compounds.

bNo data for cefepime or pip-tazo was provided for 1993-1995. 
Table 4: Cumulative fraction of response for various drug regimens against Acinetobacter spp. MICs collected from the Merck ICU Surveillance Study Program (ISS), in four time intervals.

\begin{tabular}{|c|c|c|c|c|c|}
\hline \multirow{2}{*}{$\begin{array}{l}\text { Antimicrobial agent: } \\
\text { Time interval: }\end{array}$} & \multicolumn{5}{|c|}{ CFRa (\%) } \\
\hline & $1993-1995 b$ & $1996-1998$ & $|999-200|$ & 2002-2004 & $\mathrm{p}$-value \\
\hline Imipenem 500 mg q6h & 94.5 & 91.5 & 84.8 & 84.6 & $<.0001$ \\
\hline Imipenem I g q8h & 94.2 & 92.0 & 86.2 & 86.1 & $<.0001$ \\
\hline Ceftriaxone I g q24h & 2.4 & 1.8 & 1.9 & 1.9 & .1651 \\
\hline Ceftriaxone 2 g q24h & 6.7 & 5.1 & 5.2 & 4.9 & .0005 \\
\hline Cefepime I g q I $2 \mathrm{~h}$ & - & 50.8 & 45.2 & 35.4 & $<.0001$ \\
\hline Cefepime $2 \mathrm{~g}$ q $12 \mathrm{~h}$ & - & 65.8 & 63.1 & 49.7 & $<.0001$ \\
\hline Cefepime 2 g q8h & - & 78.5 & 80.3 & 64.4 & $<.0001$ \\
\hline Pip-Tazo 3.375 g q6h & - & 56.4 & 51.3 & 43.6 & $<.0001$ \\
\hline Pip-Tazo 4.5 g q8h & - & 51.9 & 47.4 & 40.5 & $<.0001$ \\
\hline Pip-Tazo 4.5 g q6h & - & 61.0 & 55.3 & 46.6 & $<.0001$ \\
\hline
\end{tabular}

aCFRs were calculated at 40\% $\mathrm{T}$ > MIC for imipenem and 50\% $\mathrm{T}>\mathrm{MIC}$ for the other compounds.

bNo data for cefepime or pip-tazo was provided for 1993-1995.

lack thereof) in the overall group. For this reason, the study also examined two species individually: P. aeruginosa and Acinetobacter species. Both species are implicated in a variety of nosocomial infections (including pneumonia, bacteremia, skin infections and others), and reports of multi-drug resistant strains have been increasing[14]. $P$. aeruginosa was the most prevalent species in the ISS Program dataset, at $22 \%$ of tested isolates. This species is an important nosocomial pathogen not only because of its frequency, but also because it possesses intrinsic resistance to many antimicrobial agents, and has the ability to acquire both plasmid-mediated and chromosomal resistance genes[15]. Acinetobacter spp. was the fifth most prevalent pathogen in the dataset (at 6\%), but was modeled individually because high levels of resistance have been reported in Latin America and in some regions of the United States[1,16]. Multi-drug resistance in these two species is associated with increased mortality, and has become sufficiently problematic in some locations that alternative therapy using older and more toxic agents such as polymyxins is sometimes considered the best option [17-19].

Against $P$. aeruginosa, cefepime, imipenem and piperacillin-tazobactam showed statistically significant declines in CFR over time for all regimens except cefepime $2 \mathrm{~g}$ q8h. This regimen also was clearly the most potent, with predicted CFRs $>90 \%$, where every other regimen had predicted CFRs $<80 \%$ by the last time period. This suggests that, while more aggressive dosing may enable a compound to kill more reliably (i.e., with a greater probability of success), this effect no longer holds once the potency of the agent has sufficiently eroded. Overall, the magnitude of the CFR declines for all the regimens over the 12-year period of the study remains small, reflecting a steady, although moderate, upward shift in the distribution of MICs.
Against Acinetobacter spp., statistically significant declines in CFR were observed for all regimens of cefepime, imipenem and piperacillin-tazobactam. Further, the magnitude of the decline across periods is much greater than that observed against $P$. aeruginosa - on the order of $10 \%-$ $15 \%$ decline in CFR against the former species versus 2$3 \%$ versus the latter. While both declines show statistically significant trends, whether or not the low single digit declines against $P$. aeruginosa are clinically significant is debatable - that is, these declines in effectiveness are probably noticed in some institutions more than others, to the extent that the higher MICs observed here are not evenly distributed among hospitals. However, the larger declines in predicted efficacy against Acinetobacter are of great enough magnitude that a clinical effect may commonly be seen. Indeed, cefepime and piperacillin-tazobactam were predicted to have such low CFRs $(<50 \%$ for all regimens except cefepime $2 \mathrm{~g} \mathrm{q} 8 \mathrm{~h}$, with a predicted CFR of $65 \%$ ) as to be of little use clinically. Imipenem, while showing declines in CFR over the four time periods, is the only modeled agent with a high enough probability of achieving its best bactericidal effect to be relied upon clinically. These results reflect a species with rapidly increasing MICs to the modeled compounds.

\section{Conclusion}

The MIC distributions of ICU pathogens to these commonly prescribed compounds have increased significantly over time, and their effectiveness as measured by ability to attain PD targets has declined. Statistically significant declines in effectiveness were noted against $P$. aeruginosa and Acinetobacter spp. for all regimens analyzed except for ceftriaxone, which has no activity to these organisms. Against $P$. aeruginosa, cefepime $2 \mathrm{~g}$ q8h remains a potent choice for therapy; the therapeutic utility of imipenem and piperacillin-tazobactam can be maximized by aggressive dosing. Against Acinetobacter spp., declines in effec- 
tiveness are statistically (and likely clinically) significant. Among the modeled agents, only imipenem remains a viable therapeutic option against this organism; more aggressive dosing of the other compounds does not bring current effectiveness within range of earlier clinical reliability, suggesting that an impact on outcomes may be observed.

\section{Competing interests}

KJE and SL have no competing interests to declare. DPN, JPQ and GVD have received research grants from and have acted as consultants to the study sponsor. GG and MAA are employees of the study sponsor.

\section{Authors' contributions}

KJE performed the statistical analyses and drafted the manuscript. DPN conceived of the study, participated in its design, and helped to draft the manuscript. SL, JPQ and GVD contributed to the design of the study and the interpretation and analysis of data, and GVD and JPQ served as study directors. GG and MAA coordinated and executed the Intensive Care Unit Surveillance Study Program, and managed the data. All authors read and approved the final manuscript.

\section{Acknowledgements}

This study was funded by a grant from Merck \& Co., Inc., Whitehouse Station, NJ.

\section{References}

I. National Nosocomial Infections Surveillance (NNIS) System Report, data summary from January 1992 through June 2004, issued October 2004. Am J Infect Control 2004, 32(8):470-485.

2. Giamarellos-Bourboulis EJ, Papadimitriou E, Galanakis N, Antonopoulou A, Tsaganos T, Kanellakopoulou K, Giamarellou H: Multidrug resistance to antimicrobials as a predominant factor influencing patient survival. Int $J$ Antimicrob Agents 2006, 27(6):476-48I.

3. Talbot GH, Bradley J, Edwards JE Jr., Gilbert D, Scheld M, Bartlett JG: Bad bugs need drugs: an update on the development pipeline from the Antimicrobial Availability Task Force of the Infectious Diseases Society of America. Clin Infect Dis 2006, 42(5):657-668.

4. Kuti JL, Nicolau DP: Making the most of surveillance studies: summary of the OPTAMA Program. Diagn Microbiol Infect Dis 2005, 53(4):28I-287.

5. DeRyke CA, Kuti JL, Nicolau DP: Pharmacodynamic target attainment of six beta-lactams and two fluoroquinolones against Pseudomonas aeruginosa, Acinetobacter baumannii, Escherichia coli, and Klebsiella species collected from United States intensive care units in 2004. Pharmacotherapy 2007, 27(3):333-342.

6. Gillespie EL, Kuti JL, Nicolau DP: Pharmacodynamics of antimicrobials: treatment optimisation. Expert Opin Drug Metab Toxicol 2005, I(3):35I-36I.

7. DeRyke CA, Kuti JL, Nicolau DP: Reevaluation of current susceptibility breakpoints for Gram-negative rods based on pharmacodynamic assessment. Diagn Microbiol Infect Dis 2007, 58(3):337-344.

8. Mouton JW, Dudley MN, Cars O, Derendorf H, Drusano GL: Standardization of pharmacokinetic/pharmacodynamic (PK/PD) terminology for anti-infective drugs: an update. J Antimicrob Chemother 2005, 55(5):60I-607.
9. Turnidge JD: The pharmacodynamics of beta-lactams. Clin Infect Dis 1998, 27(I): 10-22.

10. Walker R, Andes D, Conklin J, Ebert SC, Craig WA: Pharmacodynamic activities of meropenem in an animal infection model [abstract], in: Program and Abstracts of the 34th Interscience Conference on Antimicrobial Agents and Chemotherapy (Orlando). Washington, DC: American Society for Microbiology 1994, 147:.

II. Jones RN, Craig WA, Ambrose PG, Dudley MN, Pottumarthy S: Reevaluation of Enterobacteriaceae MIC/disk diffusion zone diameter regression scattergrams for 9 beta-lactams: adjustments of breakpoints for strains producing extended spectrum beta-lactamases. Diagn Microbiol Infect Dis 2005, 52(3):235-246.

12. Newcombe RG: Two-sided confidence intervals for the single proportion: comparison of seven methods. Stat Med 1998, I7(8):857-872.

13. Kuti JL, Ong C, Lo M, Melnick D, Soto N, Nicolau DP: Comparison of probability of target attainment calculated by Monte Carlo simulation with meropenem clinical and microbiological response for the treatment of complicated skin and skin structure infections. Int J Antimicrob Agents 2006, 28(I):62-68.

14. Lim SM, Webb SA: Nosocomial bacterial infections in Intensive Care Units. I: Organisms and mechanisms of antibiotic resistance. Anaesthesia 2005, 60(9):887-902.

15. Waterer GW, Wunderink RG: Increasing threat of Gram-negative bacteria. Crit Care Med 200I, 29(4 Suppl):N75-8I.

16. Gales AC, Jones RN, Turnidge J, Rennie R, Ramphal R: Characterization of Pseudomonas aeruginosa isolates: occurrence rates, antimicrobial susceptibility patterns, and molecular typing in the global SENTRY Antimicrobial Surveillance Program, 1997-1999. Clin Infect Dis 200I, 32 Suppl 2:SI46-55.

17. Aloush V, Navon-Venezia S, Seigman-lgra Y, Cabili S, Carmeli Y: Multidrug-resistant Pseudomonas aeruginosa: risk factors and clinical impact. Antimicrob Agents Chemother 2006, 50(1):43-48.

18. Blot S, Vandewoude K, Colardyn F: Nosocomial bacteremia involving Acinetobacter baumannii in critically ill patients: a matched cohort study. Intensive Care Med 2003, 29(3):47| -475.

19. Falagas ME, Rafailidis PI: When To Include Polymyxins In The Empirical Antibiotic Regimen In Critically III Patients With Fever? A Decision Analysis Approach. Shock 2007, 27(6):605-609.

Publish with BioMed Central and every
scientist can read your work free of charge
"BioMed Central will be the most significant development for
disseminating the results of biomedical research in our lifetime."
Sir Paul Nurse, Cancer Research UK
Your research papers will be:
• available free of charge to the entire biomedical community
• peer reviewed and published immediately upon acceptance
• cited in PubMed and archived on PubMed Central
• yours - you keep the copyright
Submit your manuscript here:
http://www.biomedcentral.com/info/publishing_adv.asp

